\title{
The Molecular Approach in Pain Management and Improve Aesthetics Modules
}

\section{${ }^{1}$ Khalid Mohammed Alqahtani, ${ }^{2}$ Amal Radi Ayesh AlJuhani, ${ }^{1}$ Abdulrahman Mohammed - Aladhadhi , ${ }^{1}$ Mohammad Saad Alshomrani , ${ }^{3}$ Rasha Fahad AlShafi, ${ }^{4}$ Saja Hussain Majed Alsharif, ${ }^{5}$ Rawan Mohammed Natto, ${ }^{6}$ Abdulaziz Badr Abualalaa}

\author{
${ }^{1}$ King Khalid University, ${ }^{2}$ King Abdulaziz University, ${ }^{3}$ AlMaarefa Colleges for Science \& Technology, \\ ${ }^{4}$ Taibah University, ${ }^{5}$ Umm Al-Qura University, ${ }^{6}$ Imam Mohamed Bin Saud Islamic University - IMSIU
}

\begin{abstract}
:
In this review we discussed the developments in pain research and defined how the area is building on this progress to understand how pain is recorded. We likewise speculated on how these insights could lead to even more effective and specific analgesics. Methods: Computerized databases were searched for Englishlanguage controlled studies assessing molecular approach in pain management and improve aesthetics modules published to 2018. Our search was limited to only Human subject with English language. Moreover, references of identified studies were manually searched for more relevant articles. Results: Pain has been defined as an unpleasant sensory and emotional experience associated with actual or potential damage, or described in terms of such damage. It is primarily a protective mechanism designed to avoid tissue damage, is very subjective in nature with wide interpatient variability in pain sensitivity, and has been described as "a conscious experience to which the outside observer is not privy". Despite the widespread prevalence of pain, it is inadequately managed due to a lack of appropriate formal physician training that is coupled with concerns regarding the addictive liabilities of the opioids, a global phenomenon described as "opiophobia". On the other hand, patients may tolerate pain to an extent that can prevent effective treatment and result in long-term nerve damage that is frequently irreversible.
\end{abstract}

Keywords: molecular approach, pain management, aesthetics modules.

\section{Introduction:}

In the past two decades, our understanding of how the brain acquires and processes aesthetic, auditory, taste, olfactory and somatosensory information has been revolutionized. However, in nothing else sensory modality has extra fast and profound progression been made than in our comprehension of the mechanisms whereby nociceptive information is transmitted and converted in both the normal and pathological state ${ }^{[1]}$. In big part, this rapid development has actually been due to the multidisciplinary method, including the synchronized use of systems neurobiology, behavioral analysis, genes, and cell and molecular techniques that has actually been taken. Pain is needed for survival and maintaining the integrity of the organism, however sustained or chronic pain can result in second symptoms such as anxiousness and depression and in a significant reduction in the quality of life ${ }^{[2]}$. Pain pathways end in distinct brain areas that monitor the sensory and affective qualities of the starting stimulus and reveal remarkable plasticity.
Noxious stimulation always causes modifications in gene expression within the main nervous system, and different chronic pain states produce unique neurochemical signatures in the brain and spinal cord. New insights right into exactly how sensory info is processed as it relocates from the feeling organ to the cerebral cortex under the influence of a regularly transforming molecular architecture will fundamentally change the way that we come close to pain control and establish new analgesics ${ }^{[3]}$.

Pain is required for survival, however consistent pain can result in anxiousness, depression and a reduction in the quality of life. In this review we discussed the developments in pain research and defined how the area is building on this progress to understand how pain is recorded. We likewise speculated on how these insights could lead to even more effective and specific analgesics.

\section{Methodology:}


Medline and PubMed database searches were performed for articles about acute cholangitis published in English up to September 2018. The keyword search headings included assessing molecular approach in pain management and improve aesthetics modules published to 2018 were used. References list of each included study were annually searched for further relevant data.

\section{Discussion:}

\section{- Molecular signatures}

Specific patterns of phenotypic change identify different chronic pain states and it is these distinctive molecular signatures that need to be considered if effective pain control is to be attained. Pain can be taken having both sensory and affective dimensions, and is typically categorized as 'acute' or 'chronic' relying on its duration and as 'neuropathic' when it derives from straight damages to the nerve system ${ }^{[1]}$. Various pain states typically require different medicinal therapies however in some cases continue to be resistant to any sort of drug therapy. Medicines such as non-steroidal anti-inflammatories (NSAIDS) and the new cyclooxygenase-2 (COX2) inhibitors act by properly minimizing the enhanced nociceptive input from damaged tissue or from sensitized sensory neurons [1]. By comparison, systemic opiates largely act upon the main nerve system and reduce the affective or unpleasant feelings that identify pain, although local injections of opiates can additionally be effective in controlling inflammatory and neuropathic ache $[1,2]$. The challenge for molecular neurobiology is as much to discover new targets for analgesic drugs as to understand the concepts through which nociceptive information is changed. Significant progression has actually been made, much of which has actually been dependent on selective gene deletion in mice and the transgenic overexpression of specific genes. Lately, the selective ablation of discrete, biochemically specified, neuronal populations has actually begun to disclose unforeseen comprehensions right into the way that pain is processed ${ }^{[2]}$. Of equal value, nevertheless, has been the development of consistent pain models and behavioral assays to define the function these specific molecules and select populations of neurons that signal or modulate in nociceptive occasions.

- Sensory fibres : physiology and biochemistry

Sensory fibres share nociceptive and nonnociceptive details from the skin and most interior cells to the spinal cord. Sensory fibers can be separated into a large number of sub-populations [3]. Those in most cases reply to certain kinds of poisonous or non-noxious excitement. Nevertheless, after damages or inflammation, the defining characteristics of these sensory nerve cell sub-populations, such as peptide or growthfactor receptor expression, can shift and new molecules can be expressed. Damage to sensory neurons has additionally exposed the presence of 'silent' nociceptors that just end up being energetic in pathological states ${ }^{[3]}$. Anatomically, there are 2 wide groups of sensory fibers: myelinated A fibers and smaller size, unmyelinated $\mathrm{C}$ fibers. The majority of tiny size cutaneous sensory fibres- unmyelinated $\mathrm{C}$ fibers and finely myelinated A fibers- are nociceptive, although $10 \%$ of unmyelinated fibres signal just innocuous thermal info. Most of $\mathrm{C}$ fibres are polymodal nociceptors and react to all kinds of poisonous stimulation (thermal, mechanical and chemical), albeit with varying levels of sensitivity. Some years earlier, it was proposed that polymodal nociceptors can be split into two relatively nonoverlapping groups on the basis of their material of peptides or fluoride-resistant acid phosphatase (FRAP) and their mode and website of discontinuation within the spinal cord1 ${ }^{[4]}$. Extremely, this difference has actually been maintained and expanded, although some peptide-containing A fibers have currently been identified ${ }^{[3]}$. We currently know that a person group of $\mathrm{C}$ fibers (formerly determined by FRAP material) share the $\mathrm{P} 2 \mathrm{X} 3$ purine receptor, the IB4lectin-binding website and receptors for glialcell-derived neurotrophic aspect (GDNF). These fibers terminate virtually exclusively within the deeper components of the substantia gelantinosa of the spine with a glomerular kind of synaptic finishing [5]. The other teams of $\mathrm{C}$ fibers synthesizes peptides such as substance $\mathrm{P}$ and calcitonin-gene-related peptide (CGRP) and express the high-affinity nerve development variable (NGF) receptor TrkA. These fibres are greatly nociceptive and end more superficially 
within the dorsal horn ${ }^{[6]}$. Both teams of $\mathrm{C}$ fibres reply to comparable sorts of poisonous excitement and share the capsaicin VR1 receptor, which transduces noxious chemical and thermal excitement ${ }^{[7]}$.

\section{Sensory neurons as pharmacological targets}

Substantial initiative has actually been made to recognize molecules that are fundamental to signaling nociception and are selectively or preferentially expressed by nociceptive sensory neurons, as these may be new targets for analgesic drugs. Although nearly half a dozen molecules that meet these standards have been identified, two channels- the vanilloid receptor 1 (VR1) and the sensory nerve-specific (SNS) sodium channel- have actually been the most thoroughly checked out. VR1, also known as the capsaicin receptor, is a ligand-gated, nonselective cation channel expressed by the majority of smalldiameter sensory neurons ${ }^{[7]}$. VR1 responds to harmful stimulations, including capsaicin, heat and acidification, in both an easy and an integrative fashion ${ }^{[7]}$. In mice in which the gene coding for VR1 has been disrupted, level of sensitivity to noxious thermal and mechanical stimulations is normal or partially decreased, but there is a profound loss of inflammatory hyperalgesia that develops following mustard oil application or Carrageenan injection right into the paw ${ }^{[8]}$. These data suggested that $C$ fibres acutely indicate noxious chemical pain however additionally boost sensitivity to all sorts of poisonous input during the process of injury and repair service. This latest work also underlines the factor that there are parallel paths through which noxious thermal data gets to the main nervous system through the sensory nerve: via a population of fibres that express VR1, or through a second population of bigger neurons that possibly express the non-capsaicin-sensitive but vanilloid-like receptor (VRL-1), which is also sensitive to noxious heat ${ }^{[9]}$.

Sensory neurons and C fibres specifically, express numerous sodium, potassium and calcium channels. Of these channels, the SNS or outer nerve-3 (PN-3) channel is of particular attraction. Indeed, SNS is believed to be expressed preferentially by $\mathrm{C}$ fibres in rats, although some bigger neurons likewise express this channel protein. The sodium current in sensory neurons is generated by numerous voltage-gated sodium channels and these channels can be categorized according to their sensitivity to the toxic substance found in puffer fish, tetrodotoxin (TTX). In sensory neurons, at least two types of TTX-resistant channels are discovered- SNS and SNS2 (NaN)- and both appear to be revealed primarily by little sensory neurons ${ }^{[10]}$. Data indicated that both the SNS and SNS2 are involved in the transmission of nociceptive information ${ }^{[10]}$ This could be crucial considered that sodium channel overexpression has actually been observed in, and might for that reason contribute to, the extended firing that takes after neuronal damage, which is characteristic of particular neuropathic pain states. However, interruption of the Sns genetics has actually caused mice that have fairly normal harmful thermal nociception combined with a delayed growth (by 30 minutes) of inflammatory hyperalgesia. Additionally, these mice had an extensive loss of level of sensitivity to tail pressure, despite relatively normal Von Frey Threshholds measured from the hind paw [11]. This behavioral phenotype is difficult to discuss presently but may show, in part, a compensatory upregulation of other non-TTX-sensitive sodium channels.

\section{- Current Analgesic Therapy}

Three significant groups of drugs are currently utilized for the treatment of pain: NSAIDs, which include the COX preventions, aspirin, ibuprofen , indomethacin, diclofenac , and ketorolac, and acetaminophen, the mechanism of which is unidentified; the classic opioids, which include morphine and its congeners; and the analgesic adjuvants, a class of compounds that consist of antidepressants and local anesthetics that are used empirically to treat chronic pain states that have not replied to other treatments.

\section{NSAIDs.}

NSAIDs are applied in the treatment of mild to moderate pain and as an adjunct to opioids in the management of moderate to severe ache ${ }^{[12]}$. NSAIDs act by preventing the prostaglandin biosynthetic enzyme cyclooxygenase that is also called COX or PGHS (prostaglandin $\mathrm{H} 2$ synthase) and is involved in the conversion of arachidonic acid to prostaglandins $\mathrm{F}, \mathrm{D}$, and $\mathrm{E}$, prostacylin, and thromboxane ${ }^{[13]}$. The liberation of these arachidonic acid pathway products following local tissue injury contributes to outer 
sensitization and hyperalgesia. NSAIDs block prostaglandin production and therefore attenuate the outer sensitization procedure. The classic NSAIDs are very effective COX preventions, with analgesic, antiinflammatory, and antipyretic activity, and are consistently utilized to relieve the pain related to headache, tooth removal, musculoskeletal injury, RA, and OA. Their usage has actually also been expanded to cancer pain, postoperative pain, and other indications. NSAIDs have a ceiling effect in regards to their analgesic effectiveness such that complete pain relief cannot be achieved even with dosage acceleration. This is a result of their system of action that just addresses inhibition of the production of the products of the arachidonic acid pathway and thus restricts their utility to treat extreme pain. NSAIDs therefore do not block the activation of $\mathrm{C}$-fibers and have no direct impact on acute nociceptive pain. Nevertheless, aspirin might have properties along with its effects of COX inhibition that involve inhibition of neutrophil activation via actions on the cell surface area adhesion molecules (CD11b/CD18) and might be in charge of some elements of the analgesic and antipyretic activities of NSAIDs

[14]. In addition, COX inhibitors can inhibit the activity of the intracellular indicating molecule NF $\kappa B$ [15]. The negative effects related to the classic NSAIDs consist of gastrointestinal bleeding, ulceration, and perforation, inhibition of platelet aggregation, nephrotoxicity, and, in $10 \%$ of those experiencing such adverse effects, demise ${ }^{[13]}$. These adverse effects restrict their use, and NSAIDs such as ketorolac have labeling for minimal duration of use. The identification of two COX isoforms, a constitutively active enzyme (COX-1) that is involved in the production of those prostaglandins needed for typical cellular function and an inducible (COX2 ) isoform that mediates the inflammatory effects of prostaglandins, [13] recommends that these side effects might be surmountable.

\section{Opioids.}

Opioids such as morphine and codeine are used as monotherapy in the treatment of modest to extreme pain and are often added when pain is improperly controlled by NSAIDs. Opioids generate their impacts by activating receptors in the brain and spine. The opioid receptor family is a G-protein coupled receptor (GPCR) superfamily, defined by a heptahelical structural motif. Opioid receptors were designated as $\mu, \delta$, and $\kappa$ subtypes ${ }^{[16]}$ based upon the synthetic ligands originally used to categorize them but have actually been controversially relabelled OP3, OP1, and OP2 by the IUPHAR Nomenclature Committee based upon pharmacological requirements [17]. An 'orphan' member of the opioid receptor family, ORL1 has actually also been determined that is structurally similar to other members of the opioid family but is fairly insensitive to opioid agonists. The heptadecapeptide nociceptin is an endogenous ligand for the ORL1 receptor and generates hyperalgesia, protects against opioid analgesia, and produces analgesia or has no effect in rodent hot plate versions ${ }^{[18]}$. This peptide impairs spatial knowing and has actually been implicated in anxiogenic responses. It does not interact potently with $\mu, \delta$, and $\kappa$ receptors.

Amongst the adverse effects related to the clinical use of opioids are constipation due to inhibition of gut motility, a substantial side effect that is frequently underestimated and, in lots of circumstances, leads the patient to select pain over the GI adverse effects of opioids; breathing depression due to activation of opioid receptors in the breathing centers of the brain stem; dependency; and tolerance. The social and lawful concerns associated with utilize, diversion, and regulative constraints contribute to an underutilization of opioids, especially for the management of chronic nonmalignant pain ${ }^{[20]}$. Nevertheless, some physicians are willing to accept what they take into consideration a small threat in order to accomplish a level of pain control that is not attainable by various other methods. In 25000 cancer patients taking narcotics, only 7 came to be addicted ${ }^{[19]}$, a reality credited to alternate dosage forms of morphine that avoided the "rush" that causes obsession. It has also been noted that patients in extreme pain do not experience the "high" connected with opioid use in regular subjects.

\section{Analgesic Adjuvants.}

Analgesic adjuvants consist of a number of compounds that have primary therapeutic indications aside from pain control but which have been discovered in some instances, in the clinical setting, to be efficient in pain control, specifically that consisting of neuropathic pain 
[21]. Since ache has both physical and psychological components, it is possible that some types of pain that are treated with antidepressants have their etiology in the "affective motivational" element of anxiety ${ }^{[22]}$. Tricyclic antidepressants (TCAs) like amitryptiline and nortryptiline are frequently made use of when NSAID and opioid therapy have actually fallen short to supply pain relief and as first-line treatment for patients experiencing constant, burning-type pain. Adverse effects related to TCA treatment include orthostatic hypotension, sedation, dry mouth, constipation, urinary retention, glaucoma, and cognitive impairment. Because of this, treatment is initiated at low doses and titrated over numerous weeks to an efficient dose, frequently postponing the accomplishment of adequate pain relief. With the moment required to evaluate NSAIDs and opioids, patients that finally achieve pain alleviation making use of TCAs can go from 5 to 9 months or longer prior to adequate therapy is gotten. The selective serotonin reuptake preventions (SSRIs) fluoxetine and paroxetine are usually considered inefficient as analgesic adjuvants. One more SSRI, sertraline, has moderate impacts on pain limit that are dumbfounded by placebo actions ${ }^{[23]}$. These searchings' for would certainly suggest that the classical TCAs generate their analgesic activities by assisting in coming down inhibitory monoaminergic paths in the dorsal horn of the spinal cord, especially NE instead of 5HT paths ${ }^{[25]}$. Given systemically, the R2-adrenoceptor agonist, clonidine (34), can modulate neuropathic pain ${ }^{[24]}$.

\section{Conclusion:}

Pain has been defined as an unpleasant sensory and emotional experience associated with actual or potential damage, or described in terms of such damage. It is primarily a protective mechanism designed to avoid tissue damage, is very subjective in nature with wide interpatient variability in pain sensitivity, and has been described as "a conscious experience to which the outside observer is not privy".

Despite the widespread prevalence of pain, it is inadequately managed due to a lack of appropriate formal physician training that is coupled with concerns regarding the addictive liabilities of the opioids, a global phenomenon described as "opiophobia". On the other hand, patients may tolerate pain to an extent that can prevent effective treatment and result in longterm nerve damage that is frequently irreversible. Some 10-15 neurotransmitters/neuromodulators have now been identified as being involved to varying degrees in the various aspects of nociceptive pathway activity in both the spinal cord and in the ascending and descending central nervous system (CNS) pain-processing paths. These have the opportunity to supply with multiple opportunities for the development of novel analgesics distinct from the traditional NSAIDs and opioids. Molecular biology and systems neuroscience are generating a new understanding of pain and should lead to new therapeutic strategies to control pain. In many ways, we are still at the beginning and need further and deep clinical studies to add more information and knowledge on the side effects of using such new drugs in an attempt to control (reduce) systemic pains due to tissue damages as well as decreasing pain sensitivity .

\section{Reference:}

1. Fields $H$ (1997): in Molecular Neurobiology of Pain (ed. Borsook, D.) 307-317 (IASP, Seattle).

2. Yaksh T L (1999): Spinal systems and pain processing: development of novel analgesic drugs with mechanistically defined models. Trends Pharmacol. Sci., 20:329-337.

3. Snider WD and McMahon SB (1998): Tackling pain at the source: new ideas about nociceptors. Neuron., 20: 629-632.

4. Nagy JI and Hunt SP(1982): Fluorideresistant acid phosphatasecontaining neurons in dorsal root ganglia are separate from those containing substance $\mathrm{P}$ or somatostatin. Neuroscience, 7: 8997.

5. Bennett DL, Michael GJ, Ramachandran N et al. (1998): A distinct subgroup of small DRG cells express GDNF receptor components and GDNF is protective for these neurons after nerve injury. J. Neurosci., 18, 30593072.

6. Ribeiro-da-Silva A, and Coimbra A (1984): Capsaicin causes selective damage to type I synaptic glomeruli in rat 
substantia gelatinosa. Brain Res., 290:380-383.

7. Caterina MJ, Schumacher MA, Tominaga M et al. (1997): The capsaicin receptor: a heatactivated ion channel in the pain pathway. Nature, 389(6653):816-824.

8. Caterina MJ, Leffler A, Malmberg AB et al. (2000): Impaired nociception and pain sensation in mice lacking the capsaicin receptor. Science, 288(5464):306-313.

9. Caterina MJ, Rosen TA, Tominaga M et al. (1999): A capsaicin-receptor homologue with a high threshold for noxious heat. Nature,398(6726):436-441.

10. Waxman SG, Dib-Hajj S, Cummins TR et al. (1999): Sodium channels and pain. Proc. Natl Acad. Sci., 96(14): 7635-7639.

11. Akopian NA, Souslova V, England S et al. (1999): The tetrodotoxin-resistant sodium channel SNS has a specialized function in pain pathways. Nature Neurosci., 2(6):541-548.

12. Levine JD (1998): New directions in pain research: molecules to maladies. Neuron, 20: 649-654.

13. Vane JR, Bakhle YS, Botting RM (1998): Cyclooxygenases 1 and 2. Annu. Rev. Pharmacol. Toxicol., 38:97-120.

14. Cronstein NB and Weissman G (1995): Targets for antiinflammatory drugs. Annu. Rev. Pharmacol. Toxicol.,35:449462

15. Kopp $E$ and Ghosh $S$ (1994): Inhibition of NF $\kappa B$ by sodium salicyate and aspirin. Science ,265: 286-290.

16. Ossipov MH, Malan TP, Lai J et al. (1997): Opioid pharmacology of acute and chronic pain. In The Pharmacology of Pain. Handbook Exp. Pharmacol; Dickenson AH, Besson JM, Springer Verlag: Berlin; pp 306-333.

17. Dhawan BN, Cesselin F, Raghubir R et al. (1996): International classification of opioid receptors. Pharmacol. Rev., 48:567-583.

18. Henderson $G$ and McKnight AT (1997): The orphan opioid receptor and its endogenous ligand - nociceptin/ orphanin FQ. Trends Pharmacol. Sci., 18:293-300.

19. Brownlee S and Schrof JM (1997): The quality of mercy. U.S. News World Rep., 17:54-67.

20. Cleeland CS (1998): Recommendations for pain management. JAMA., 279:19141915.

21. Portenoy RK (1996): Nontraditional analgesics in the management of cancer pain. In Pain 1996-An Updated Review; Campbell JN, IASP Press: Seattle WA, pp 559-566.

22. Yaksh TL (1998): Antidepressants as analgesics. In Anesthesia: Biologic Foundations; Yaksh TL, Lynch C, Zapol WM, Maze M, Biebuyck JF, Saidman LJ, LippincottRaven: Philadelphia, pp 987997.

23. Alberts KR, Bradley LA, Alarcon GS et al. (1998): Sertraline hydrocholoride (Zoloft) alters pain threshold, sensory discrimination ability, and functional brain activity in patients with fibromylagia (FM). Abstracts American Pain Society 17th Annual Meeting, San Diego CA, pp 811.

24. Kayser V, Desmeules J, Guilbaud G (1995): Systemic clonidine differentially modulates the abnormal reactions to mechanical and thermal stimuli in rats with peripheral mononeuropathy. Pain, 60:275-285.

25. Millan MJ (1997): The role of descending noradrenergic and serotoninergic pathways in the modulation of nociception: focus on receptor multiplicity. In The Pharmacology of Pain. Handbook Exp. Pharmacol.; Dickenson AH, Besson JM, Springer Verlag: Berlin, pp 385-446. 